\title{
Establishing gene models from the Pinus pinaster genome using gene capture and BAC sequencing
}

Pedro Seoane-Zonjic ${ }^{1}$, Rafael A. Cañas', Rocío Bautista1, Josefa Gómez-Maldonado', Isabel Arrillaga², Noé Fernández-Pozo ${ }^{3}$, M. Gonzalo Claros ${ }^{1}$, Francisco M. Cánovas ${ }^{1}$ and Concepción Ávila ${ }^{1 *}$

\begin{abstract}
Background: In the era of DNA throughput sequencing, assembling and understanding gymnosperm mega-genomes remains a challenge. Although drafts of three conifer genomes have recently been published, this number is too low to understand the full complexity of conifer genomes. Using techniques focused on specific genes, gene models can be established that can aid in the assembly of gene-rich regions, and this information can be used to compare genomes and understand functional evolution.
\end{abstract}

Results: In this study, gene capture technology combined with BAC isolation and sequencing was used as an experimental approach to establish de novo gene structures without a reference genome. Probes were designed for 866 maritime pine transcripts to sequence genes captured from genomic DNA. The gene models were constructed using GeneAssembler, a new bioinformatic pipeline, which reconstructed over $82 \%$ of the gene structures, and a high proportion ( $85 \%$ ) of the captured gene models contained sequences from the promoter regulatory region. In a parallel experiment, the $P$. pinaster BAC library was screened to isolate clones containing genes whose CDNA sequence were already available. BAC clones containing the asparagine synthetase, sucrose synthase and xyloglucan endotransglycosylase gene sequences were isolated and used in this study. The gene models derived from the gene capture approach were compared with the genomic sequences derived from the BAC clones. This combined approach is a particularly efficient way to capture the genomic structures of gene families with a small number of members.

Conclusions: The experimental approach used in this study is a valuable combined technique to study genomic gene structures in species for which a reference genome is unavailable. It can be used to establish exon/intron boundaries in unknown gene structures, to reconstruct incomplete genes and to obtain promoter sequences that can be used for transcriptional studies. A bioinformatics algorithm (GeneAssembler) is also provided as a Ruby gem for this class of analyses.

Keywords: BAC, Bioinformatic pipeline, Gene capture, Gene model construct, Gene structure, Maritime pine, Promoter studies

\footnotetext{
* Correspondence: cavila@uma.es

'Departamento de Biología Molecular y Bioquímica, Facultad de Ciencias,

Universidad de Málaga, Campus de Teatinos s/n, E-29071 Málaga, Spain

Full list of author information is available at the end of the article
} 


\section{Background}

Forests ecosystems play a fundamental role in the regulation of terrestrial carbon sinks and represent nearly $80 \%$ of the world's total plant biomass [1]. Conifers dominate a large part of the forests in the northern hemisphere, and they are intensively exploited as the primary source of wood for industrial purposes [2]. Conifers also exhibit unique characteristics among vascular plants, including: high genetic variability, long half-lives, seasonal survival, adaptation to secondary growth, and wood deposition among others [3]. Despite their economic and ecological importance, genomic studies of conifers have been hampered by the large size of their genomes, which range from 20 to $40 \mathrm{~Gb}$, approximately 200 times the size of the Arabidopsis genome and approximately seven times the size of human genome [4]. However, recent technical advances in genomic sequencing have enabled the assembly of the Norway spruce [5], white spruce [6] and loblolly pine [7] genomes, and the sequencing of a number of additional species is underway $[4,8]$. Although these assemblies represent landmark in conifer genomics, technological challenges continue to face the assembly and annotation of conifer genomes; they are characterized by a proliferation of retrotransposons, highly diverged repetitive sequences, accumulation of non-coding regions and extensive gene duplication $[4,8]$. Also, large families of transposons and retrotransposons have been reported to occupy long stretches of the sequences in Pinus genomes $[8,9]$.

The analysis of BAC clones has been the most common approach used for genome characterization and in hierarchical sequencing projects, such as the human genome [10] or other genomes without available references [11]. The screening of BAC libraries has been used to target gene-rich regions in white spruce, but the approach has proven to be very laborious because most clones contain the non-coding regions of the genes, which is expected due to the large size of conifer genomes [12].

An alternative to obtaining the gene sequences of large and complex genomes is to perform an enrichment step to isolate the genomic DNA sequences of interest that contain the coding regions of genes by massive parallel sequencing and use them for further analysis [13]. This system named "gene capture", uses rapid selective hybridization technique to obtain sequences of interest much more efficiently [14]. "-Gene capture-" has been widely used as a diagnostic tool for human whole exome analyses [15-17] but the use of the technique in plants has been much more limited $[18,19]$.

In this work, we used "-gene capture-" to elucidate the target, gene-rich regions in the genome of the maritime pine (Pinus pinaster L. Aiton), a conifer species of great ecological and economic importance in Europe and for which whole-transcriptome resources are available [20, 21]. To achieve this goal, 120-mer probes were designed from 866 tentative maritime pine transcripts, which include the probes for three characterized BAC clones as a control. These BAC clones were isolated by screening a maritime pine BAC library using specific cDNA probes [22] and then used as a reference for gene capture assays.

In this approach, megagametophyte calli haploid DNA from maritime pine was isolated, fractioned and bounded by a series of specific adapters for 454 sequencing. The captured genomic sequences were sequenced in an FLXTitanium platform, and the reads were assembled and analyzed using the GeneAssembler bioinformatic pipeline to recover the gene models. This experimental approach also provided sequences for the proximal promoter region of the targeted genes. This can be used as initial information for genome walking to thoroughly characterize the cis elements contained in the regulatory region of these genes.

\section{Results \\ BAC clone isolation and characterization}

A Pinus pinaster BAC library that had been previously established in pools [22] was used to screen for particular clones containing gene coding sequences for asparagine synthetase 1 (AS1) [23] cDNA [GenBank: HQ625490], sucrose synthase (SuSy) cDNA [GenBank: AJ309093] and xyloglucan endotransglycosylase (XET) cDNA [GenBank: FN824804]. A detailed protocol for the identification and isolation of these BAC clones is described in the Methods section and summarized in the Additional file 1: Figure S1. As described in the Methods section, DNA was prepared from the purified BAC clones and fully sequenced, and the sequence assemblies for AS1, SuSy and XET were deposited in GenBank [GenBank: KP172187, GenBank: KP172194 and GenBank: KP172185 respectively]. Figure 1 depicts the corresponding BAC clone structures as single scaffolds. The sequences of the BAC clones were annotated and used to visualize the gene structure using GENote v. $\beta$. [23], which was used to detect the presence of the gene, its promoter location, the putative intron-exon pattern and the presence of transposable elements. The ASI sequence in the BAC clone exactly matches the previously characterized maritime pine AS1 cDNA [24], and Fig. 1a shows the pattern of the BAC clone containing the AS1 gene assembled in a single scaffold of $46,111 \mathrm{bp}$. The AS1 gene is organized into 14 exons spanning a region of 3974 nucleotides, and the processed length without introns corresponds to a gene product with 1782 nucleotides that yields a protein with 593 amino acids. By comparing the Arabidopsis and poplar $A S$ sequences in the databases, we determined the exon and intron structures, which are presented in Tables 1 and 2, respectively. The size and 


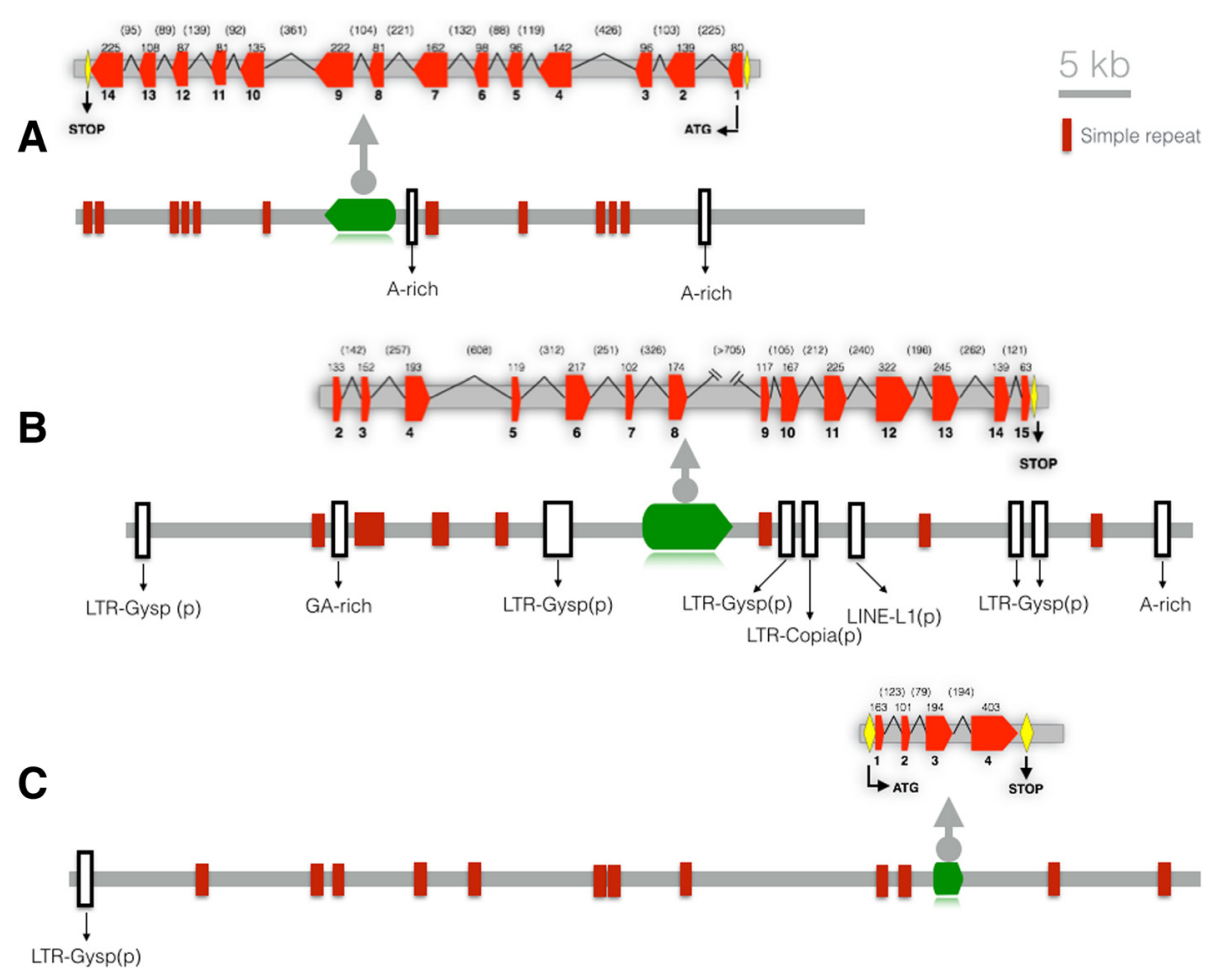

Fig. 1 Structure of maritime pine genomic DNA contained in the BAC clones GenBank: KP172187 (a), GenBank: KP172184 (b) and GenBank: KP172195 (c) respectively. Light red boxes represent exons and introns the intervening line. The length in base pairs of each intron and exon is also indicated. Segments with similarity to transposable element and repetitive regions were identified with Repeat Masker and are represented by white and dark red boxes. The scale bar represents $5 \mathrm{kbp}$.

Table 1 Exon length comparisons among complete AS genes from $P$. pinaster and AS from two angiosperm plants

\begin{tabular}{|c|c|c|c|c|c|c|}
\hline $\begin{array}{l}\text { Exon } \\
\text { length (nt) }\end{array}$ & $\begin{array}{l}\text { Pinus pinaster } \\
\text { AS1/AS3/AS5 }\end{array}$ & ASN_BAC & $\begin{array}{l}\text { Arabidopsis thaliana } \\
\text { ASN3 (At5g10240) }\end{array}$ & $\begin{array}{l}\text { Arabidopsis thaliana ASN1 } \\
\text { (At3g47340) }\end{array}$ & $\begin{array}{l}\text { Populus trichocarpa AS } \\
\text { (Potri.009G072900) }\end{array}$ & $\begin{array}{l}\text { Populus trichocarpa AS } \\
\text { (Potri.005G075700) }\end{array}$ \\
\hline E1 & 80 & 80 & 80 & 80 & 80 & 80 \\
\hline E2 & 139 & 139 & 139 & 139 & 139 & 139 \\
\hline E3 & 96 & 96 & 100 & 96 & 96 & 96 \\
\hline E4 & 142 & 142 & 135 & 333 & 142 & 142 \\
\hline E5 & 96 & 96 & 96 & & 191 & 96 \\
\hline E6 & 98 & 98 & 98 & & & 95 \\
\hline E7 & 162 & 162 & 162 & 162 & 162 & 162 \\
\hline E8 & 81 & 81 & 84 & 303 & 81 & 81 \\
\hline E9 & 222 & 222 & 222 & & 222 & 222 \\
\hline E10 & 135 & 135 & 129 & 135 & 135 & 135 \\
\hline E11 & $81 / 84^{*}$ & 81 & 81 & 168 & 81 & 81 \\
\hline E12 & 87 & 87 & 87 & & 87 & 87 \\
\hline E13 & 108 & 108 & 108 & 108 & 108 & 108 \\
\hline E14 & $252 / 243 / 240$ & 252 & 78 & 231 & 246 & 231 \\
\hline
\end{tabular}


Table 2 Intron length comparisons among complete AS genes from P. pinaster and AS from two angiosperm plants

\begin{tabular}{|c|c|c|c|c|c|c|c|c|}
\hline $\begin{array}{l}\text { Intron } \\
\text { length (nt) }\end{array}$ & $\begin{array}{l}\text { Pinus } \\
\text { pinaster } \\
\text { AS1 }\end{array}$ & $\begin{array}{l}\text { Pinus } \\
\text { pinaster } \\
\text { AS3 }\end{array}$ & $\begin{array}{l}\text { Pinus } \\
\text { pinaster } \\
\text { AS5 }\end{array}$ & ASN_BAC & $\begin{array}{l}\text { Arabidopsis thaliana } \\
\text { ASN3 (At5g10240) }\end{array}$ & $\begin{array}{l}\text { Arabidopsis thaliana } \\
\text { ASN1 (At3g47340) }\end{array}$ & $\begin{array}{l}\text { Populus trichocarpa AS } \\
\text { (Potri.009G072900) }\end{array}$ & $\begin{array}{l}\text { Populus trichocarpa AS } \\
\text { (Potri.005G075700) }\end{array}$ \\
\hline 11 & 225 & 213 & 124 & 225 & 260 & 422 & 509 & 78 \\
\hline 12 & 101 & 88 & 112 & 101 & 186 & 88 & 156 & 227 \\
\hline 13 & 426 & 136 & $422^{*}$ & 426 & 192 & 82 & 152 & 369 \\
\hline 14 & 119 & 133 & 126 & 119 & 102 & 78 & 94 & 263 \\
\hline 15 & 88 & 112 & 117 & 88 & 184 & & 115 & 520 \\
\hline 16 & 132 & 213 & 139 & 132 & 94 & & & 721 \\
\hline 17 & 219 & 261 & 221 & 219 & 85 & 136 & 89 & 533 \\
\hline 18 & 104 & 104 & 103 & 104 & 84 & 80 & 93 & 81 \\
\hline 19 & 87 & 87 & 87 & 87 & 83 & & 96 & 136 \\
\hline 110 & 92 & 89 & 99 & 92 & 209 & 83 & 120 & 453 \\
\hline 111 & 139 & 100 & 109 & 139 & 85 & 91 & 85 & 110 \\
\hline 112 & 89 & 104 & 102 & 89 & 91 & & 91 & 236 \\
\hline 113 & 95 & 95 & 90 & 95 & 96 & 93 & 100 & 152 \\
\hline
\end{tabular}

*Incomplete Intron. The gene capture model is also included

number of exons contained in the $\mathrm{BAC}$ clone are closer to those of the ASN3 (At5g10240) gene from Arabidopsis. A total of 13 introns were identified, and their relative positions with respect to the coding region are well conserved compared to the Arabidopsis ASN3 gene as well as the $P$. trichocarpa Potri.005G075700 model (Table 2).

A similar analysis of the structure of the SuSy and $X E T$ genes was performed. The SuSy BAC clone is represented in a single scaffold of 59,327 bp (Fig. 1b), and the gene sequence is organized into 14 exons encoding a protein with 769 amino acids. The comparison to the SuSy gene models in other plants indicated that the $5^{\prime}$ end of the gene is missing in the pine BAC clone obtained for this study. The lengths and composition of the exon and intron are presented in Additional file 2: Table S1 and Additional file 3: Table S2, respectively, and a comparative study showed a high degree of conservation in the number and size of the exons in the maritime pine BAC model compared with the SuSy2 gene from Arabidopsis and the POPTRDRAFT_830445 gene from $P$. trichocarpa. In contrast, substantial differences in the number and lengths of the exons were found when compared with SuSy3 from Arabidopsis and POPTRDRAFT_826368 from Populus.

The XET BAC clone is represented in a single, 64,767-bp scaffold (Fig. 1c), and the sequence renders a model organized into 4 exons and 3 introns encoding a protein with 287 amino acids. The lengths and distribution of the exons and introns of the gene contained in the BAC clone are represented in Additional file 4: Table S3 and Additional file 5: Table S4, respectively. Two Arabidopsis gene models, XET 9 (At4g032210) and XET 3 (At3g25050), were closest to the pine gene sequence, but it was impossible to determine which is more similar to the gene contained in the pine BAC.

Considering that conifer genomes are characterized by retrotransposon proliferation and extensive regions containing repetitive elements, we performed a deeper study of the presence of these elements in the BAC clones. Through a comparison with the corresponding BACcontaining-gene available in Picea glauca (no BAC corresponding to $X E T$ was available), we analyzed the presence of repetitive elements. The content and type of the repetitions in both conifer BACs are summarized in Table 3. The percentage of bases that are part of the retroelements is quite similar in both SuSy BACs. But this percentage is significantly lower in the $A S$ pine BAC, probably due to the different lengths of both BAC clones. In terms of the types of repeated elements, the retroelements, LTRs and Gypsy/Dir1 are the most represented in both SuSy BACs as well as in the P. glauca AS BAC with similar percentages of the total number of bases. DNA transposons, Simple repeats and low complexity elements contribute less to the size of the BAC.

\section{Genomic DNA capture and gene model generation}

At the same time as the BAC library screening, we conducted a gene capture procedure using maritime pine haploid DNA [25]. Genomic DNA captured obtained using the SureSelect kit was sequenced in 454/Roche, and a total of 2,036,142 captured raw reads with an average size of 769 nt were cleaned with SeqTrimNext, producing $1,942,057$ useful reads. These reads were assembled with MIRA3, yielding a total of 144,707 contigs and 305,396 "debris" reads. The contigs served to reduce the sequence 
Table 3 Content and type of repeats present in SuSy and AS1 BACs from $P$. glauca and $P$. pinaster

\begin{tabular}{|c|c|c|c|c|}
\hline & Susy P. glauca & Susy P. pinaster & AS P. glauca & AS $P$. pinaster \\
\hline GeneBank: & KC860252 & KP172192 & KC860234 & KP172187 \\
\hline Total length: & $137047 \mathrm{bp}$ & $59397 \mathrm{bp}$ & $130154 \mathrm{bp}$ & $46111 \mathrm{bp}$ \\
\hline \multirow[t]{2}{*}{ GC level: } & $38.66 \%$ & $39.03 \%$ & $38.17 \%$ & $34.80 \%$ \\
\hline & \multicolumn{4}{|c|}{ Number of elements/percentage of sequence } \\
\hline Bases masked: & 10259 bp (7.49\%) & 5173 bp (8.71 \%) & 7548 bp (5.80 \%) & $595 \mathrm{bp}(1.29 \%)$ \\
\hline Retroelements & $7(6,78 \%)$ & $7(7,52 \%)$ & $9(4,65 \%)$ & 0 \\
\hline LINEs: & 0 & $1(0,70 \%)$ & $2(0,16 \%)$ & 0 \\
\hline L1/CIN4 & 0 & $1(0,70 \%)$ & $2(0,16 \%)$ & 0 \\
\hline LTR elements: & $7(6,78 \%)$ & $6(6,82 \%)$ & $7(4,49 \%)$ & 0 \\
\hline Ty1/Copia & $1(1,09 \%)$ & $1(0,24 \%)$ & $1(0,55 \%)$ & 0 \\
\hline Gypsy/DIRS1 & $6(5,60 \%)$ & $5(6,58 \%)$ & $6(3,94 \%)$ & 0 \\
\hline DNA transposons & 0 & 0 & $2(0,10 \%)$ & 0 \\
\hline Simple repeats: & $18(0,67 \%)$ & $17(0,94 \%)$ & 18 (0,69 \%) & $11(0,98 \%)$ \\
\hline Low complexity: & $1(0,03 \%)$ & $3(0,25 \%)$ & $7(0,36 \%)$ & $2(0,31 \%)$ \\
\hline
\end{tabular}

space and to provide longer consensus sequences to facilitate the building of the gene model.

The GeneAssembler pipeline was developed to build the most complex gene model that could code for each of the 866 selected full-length cDNAs from the pine transcriptome described in [20]. Starting with 144,707 contigs, the following steps were included: (i) gene assignment and contig filtering (Fig. 2a), (ii) contig clustering by gene (Fig. 2b) and (iii) gene model building to produce a gene model for the selected full-length proteins (Fig. 2c).

The best gene model is as follows: (i) the one that recovers more full-length seeding sequences; (ii) the one with the lowest overlap percentage of contigs at the exon level; and (iii) the one with the lowest fragmentation index, that is, the one obtained from the fewest number of contigs. The GeneAssembler gene model for each fulllength protein was saved in a gff3 file, which included the

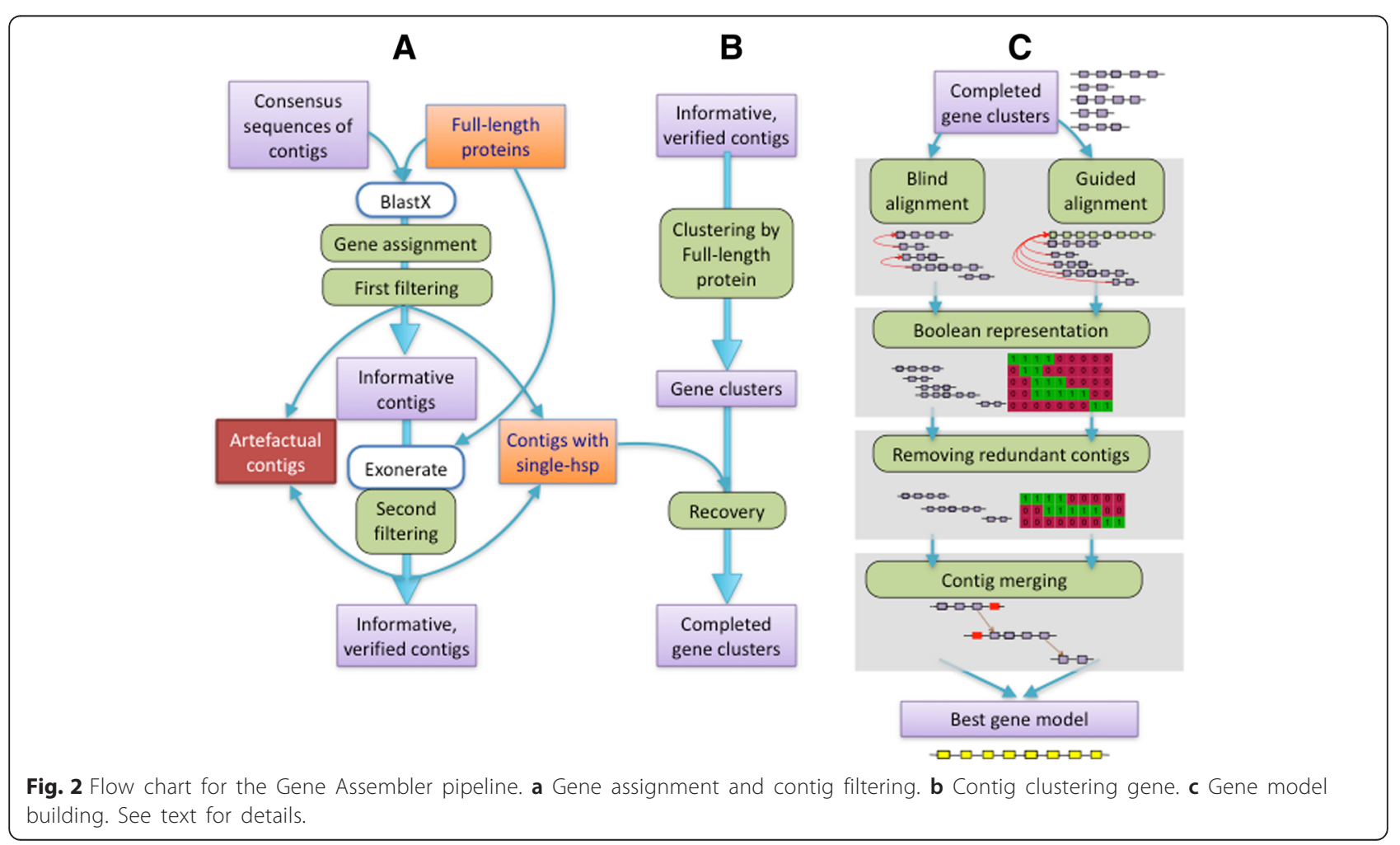


contigs. Additionally, a FASTA file was generated with the gene model sequences and an index relating each contig with its full-length protein.

Genomic sequences were recovered for the 866 maritime pine genes selected to design the probes, and this recovery was independent of the use of a reference genome for building the gene model. Accordingly, the reconstruction that was done without a reference genome was selected as it represents the highest mean recovery of each protein, approximately $82 \%$, and the lowest sequence redundancy. Most of the gene models with a high recovery percentage had between one to five exons, and particularly for those with one and two exons, there were many gene models with a recovery rate above $50 \%$. In addition, there were 20 gene models with a recovery rate over $100 \%$, but these were considered to be incorrect due to the presence of sequence repeats, which the algorithm was unable to handle. All of the generated contigs and models are available in the Pine Gene Capture database (PGC, http://www.scbi.uma.es/pgc/).

Because there is increasingly more information available about plant genomes, it is possible to make broad comparisons among them. Although it has been suggested that organisms with small genomes have smaller introns, studies of angiosperms indicate that this is not necessarily true in plants [26, 27]. We performed an analysis of the size of the introns for our 866 reconstructed maritime pine models and compared the results to homologs in the moss Physcomitrella patens and three well-characterized angiosperm genomes: Arabidopsis thaliana, Oryza sativa and Populus trichocarpa (Fig. 3). The average length of the individual introns (in bp) was 197, 98, 143, 159 and 155 for P. patens, A. thaliana, O. sativa, $P$. trichocarpa and $P$. pinaster, respectively. The average intron length did not vary significantly between $P$. pinaster and the other four models chosen for this study (Fig. 3). However, the intron lengths were more heterogeneous in O. sativa and P. trichocarpa than in the other three species considered, at least for the 866 genes included in this study. Maritime pine did not appear to have a significantly larger intron.

\section{Comparison of BAC and gene capture approaches to defining maritime pine gene models}

To further explore the quality of the genomic sequences derived from the gene capture approach, the structures of the AS, SuSy and XET genes were compared to those derived by BAC clone sequencing.

Tables 1 and 2 show the distribution of exons and introns, respectively, for the $A S$ gene obtained by BAC sequencing and that obtained by gene capture. After the analysis of the gene capture contigs, four different $A S$ gene models were found to be similar to $A S 1$, including itself; the new sequences were named $A S 3, A S 4$ and

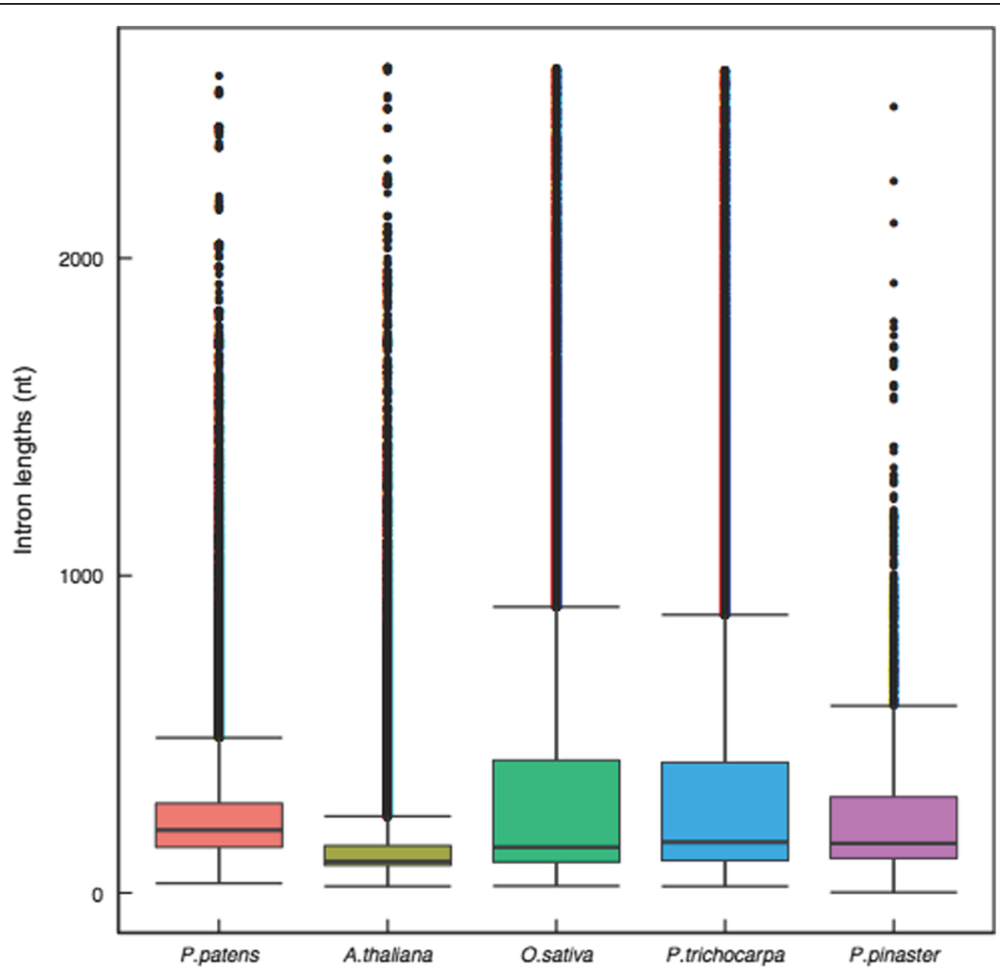

Fig. 3 Distribution of individual lengths of introns smaller than $2600 \mathrm{nt}$ using the 866 reconstructed gene models in $P$. pinaster and their orthologs from P. patens, A. thaliana, O. sativa and P. trichocarpa. The box plot includes the median values as well as outlier lengths. 
AS5. While cDNA contigs for the AS3 and AS4 genes were found in SustainPineDB (sp_v3.0_unigene3120 and sp_v3.0_unigene97582 respectively) [20], no contigs for AS5 were found in SustainPineDB or Genbank. However, the genomic sequences for the $A S 5$ ortholog are present in the Pinus taeda genome [PtAS5, Congenie:lcl|scaffold870050.1]. Figure 4 presents the structures of the five $A S$ genes of $P$. pinaster, with their relative exon/intron sizes. The AS1 and AS3 gene structures were completed, and the AS5 gene sequence was almost completed except for a part of the third intron. However, the AS4 structure was not completed due to a lack of information for the $3^{\text {rd }}$ to the $8^{\text {th }}$ introns. The gene capture assay also included probes for $A S 2$, and the sequence was independently analyzed with respect to $A S 1$. No crossovers were observed between $A S 1$ and AS2 in the gene model building process, but the gene model for $A S 2$ was incomplete as it lacked information for the $5^{\text {th }}$ to $11^{\text {th }}$ introns. The gene capture model that was generated fits to the AS3 gene as well as to AS1 and AS5. Although the comparison of the gene capture and BAC data indicates that the exon lengths and distributions from both methods agree well, the structure of the $A S$ gene in the BAC clone was identical to AS1 gene model based on the gene capture. This provides additional confirmation that the isolated BAC contained a genomic fragment encoding the maritime pine AS1 protein [28].

The gene capture model for the SuSy gene agreed well with the structure of the BAC clone. The maritime pine model is closer to the SuSy2 gene from Arabidopsis and Populus with very well conserved number, length and exon position (Additional file 2: Table S1). Only exons 7 and 15 displayed small differences of 6 and $27 \mathrm{nt}$, respectively. The combination of BAC sequencing and gene capture allowed us to complete the first exon sequence, which can be considered an additional advantage of combining the two strategies.

The BAC clone and the gene capture model displayed very similar intron sizes; even intron 8 , which contained a gap in the BAC assembly, was completed using the gene capture data (Additional file 3: Table S2). Although the distribution and length of exons was well conserved, an increase in the variability of the intron size was observed, which was similar to what was found for the $A S$ gene family. In this case, the average size of the introns of the maritime pine gene was higher than for those genes of the other two species included in the comparison.

In the third part of this study, building the XET models, we did not include poplar genes, as we did for the AS1 and SuSy genes. The XET-related gene family is very large in plants, and as the poplar genome recently underwent a whole genome-wide duplication [29], the number of XET genes to compare to find the closest model to the pine gene would be very large. Therefore, considering the larger size of the gene family, we could not obtain a single model through gene capture. Instead, the two potential gene models that were generated are listed in Additional file 4: Table S3.

\section{Gene capture of regulatory regions}

An additional aim of this study was to test if the gene capture methodology could be extended to recover unknown sequences of the $5^{\prime}$ end of the genes so that these sequences could be used: i) for a genome walking approach to obtain the promoter sequence or ii) directly for functional studies using the proximal promoter region of the genes.

To obtain the $5^{\prime}$ end of the captured genes, contigs containing the first exon and having at least $100 \mathrm{nt}$ upstream of the first exon were selected. Based on these standards, we obtained the frequency distribution of the contigs containing a noticeable upstream region of the genes (Fig. 5). The estimation from 866 gene models used in the gene capture approach was that 737 gene models contained a noteworthy 5 'region sequence. Of those, 480 had a 5 'region ranging 500 to 1000 nucleotides.

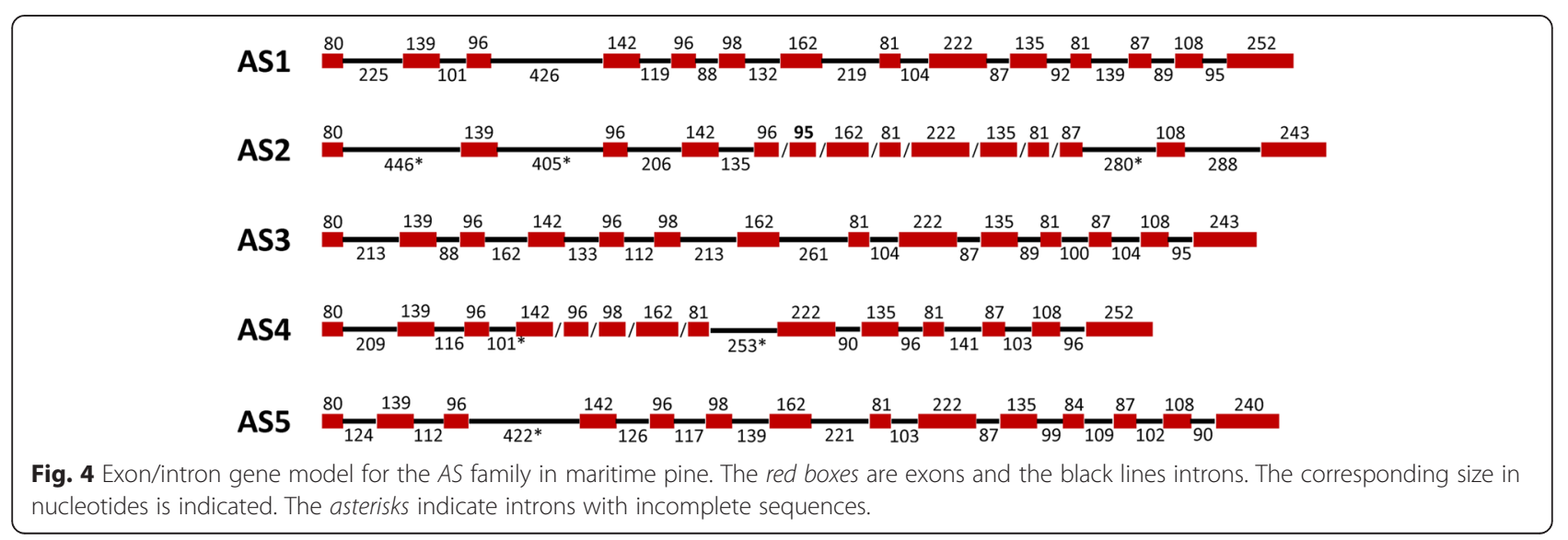




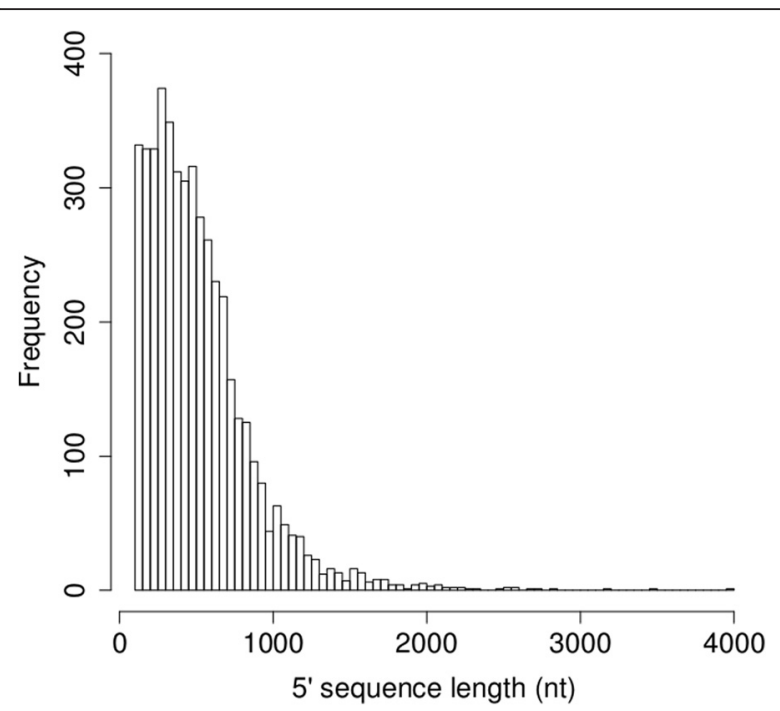

Fig. 5 Frequency distribution of contigs containing the 5'upstream region of the gene models generated. The sequence length recovered is expressed in nucleotides. The distribution was built using the contigs containing the first exon where the amino acid 10 or previous is present and where from the beginning of the first exon have at least $100 \mathrm{nt}$ upstream.

To validate these results, the 5 'upstream sequences of three genes included in our gene capture study were compared to the corresponding gene promoter sequences that had been previously cloned and functionally characterized $[30,31]$. For the glutamine synthetase (GS1a) gene, 518 nucleotides of the sp_v1.1_unigene26377 gene capture model overlapped with the GenBank promoter sequence [GenBank:AJ225121] (Additional file 6: Figure S2); 907 nucleotides for the phenylalanine ammonia lyase (PAL) gene promoter [GenBank:HE866754] overlapped with the sp_v1.1_unigene15094 model (Additional file 7: Figure S3); and 630 nucleotides for the prephenate aminotransferase (PAT) gene promoter [GenBank:HE866755] matched the unigene all_rep_c3941_PAT gene capture model (Additional file 8: Figure S4).

\section{Discussion}

In this study, we present a strategy to generate maritime pine gene models, confirmed by the structure of available BAC clones, which demonstrates that gene capture is a powerful technology for establishing gene structures in species without reference genomes, such as the maritime pine.

\section{Maritime pine gene structure}

Generally, it is well accepted that a positive relationship exits between genome size and intron lengths in eukaryotes. In conifers, which have large genomes ranging from 18 to $35 \mathrm{Gbp}$, longer introns can contribute to large genome sizes [12]. In this study, we performed a deep comparison of exon/intron distribution for three pine genes against the Arabidopsis and poplar sequences from the databases.

The intron lengths for the $A S 1$ gene (Table 2) are similar to those described for ASN1 and ASN3 in Arabidopsis, with the exception of introns 3 and 9, but none were greater than $426 \mathrm{bp}$, the length of intron 3 in the AS1 gene. In fact, when the $A S 1$ intron sizes were compared to the poplar gene models, the medium intron size was larger in poplar than in Arabidopsis or pine (Fig. 6a). When intron size is compared in the structure of the SuSy gene, the distribution is also well conserved among the pine BAC, Arabidopsis SuSy2 and poplar POPTRDRAFT_830445 genes. However, intron length is most variable among the three species, with longer introns for the maritime pine SuSy gene (Fig. 6b). For the XET gene, no significant differences were found in the size, distribution, number or length of the intron when compared to the Arabidopsis gene models. Moreover, a broad comparison of the intron sizes for the 866 gene models generated in this study (Fig. 3) showed that the medium intron size is in the range of that observed for other angiosperms models [18] (for more details, see gene models in the Pine Genome Capture Database PGCD: http://www.scbi.uma.es/pgc/).

The second feature contributing to the large conifer genomes is retrotransposon expansion, which contrasts with what has been described for large angiosperm genomes, where gene duplications and, polyploidization, as well as retrotransposons expansion, are the most common features contributing to genome size [32, 33]. Thus, retrotransposon expansion can be of primary importance in explaining genome size in Pinus species [34]. We analyzed the presence of retroelements in the BAC clones included in this study by comparing two available BACs containing the AS1 and SuSy genes in P. glauca.

In terms of number/percentage, the LTR and Gypsy retrotransposons are most abundant in the SuSy and AS1 BACs from P. glauca and P. pinaster (Table 3). These findings suggest that retrotransposon expansion is a reasonable hypothesis to explain the large genome size in $P$. pinaster, as has been proposed for other conifers $[34,35]$. However, much more data on the retrotransposon families of maritime pine are needed to confirm this hypothesis.

\section{Gene capture results are consistent with BAC clone} structures; this outcome can be extended to other frames The algorithm developed for building gene models in this work has been used with plants as target organisms, but it is not restricted to them, its use with gene capture data derived from other organisms is possible.

A total of 866 gene models were obtained with our inhouse bioinformatics pipeline (GeneAssembler), with a 

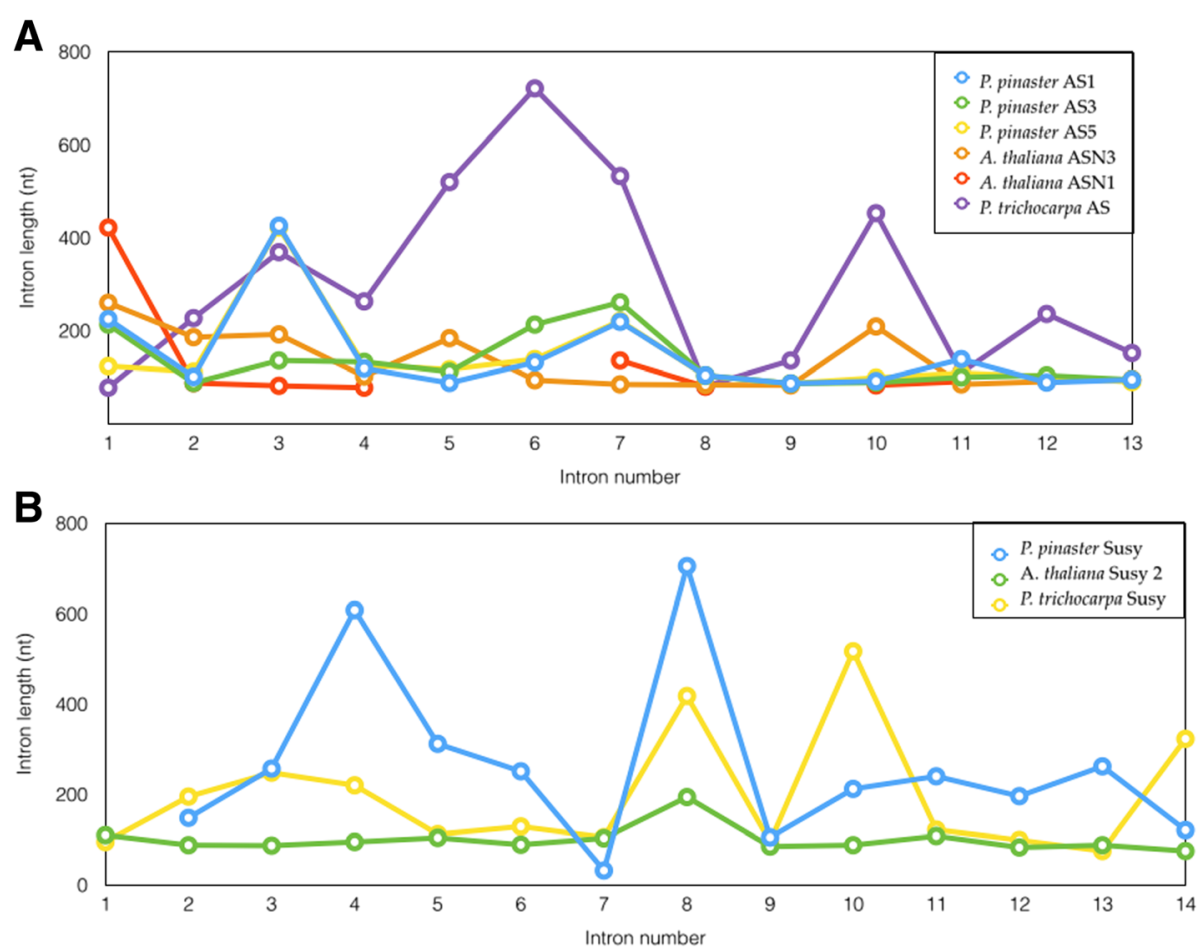

Fig. 6 Representation of gene intron size: (a) AS genes in P. pinaster, A. thaliana and P. trichocarpa; (b) SuSy gene from P. pinaster, A. thaliana and P. trichocarpa. On the $X$ axis is showed the position of the introns and on the $Y$ axis is showed the length of introns in nucleotides.

median recovery percentage of $82 \%$ from the original full-length sequence. However, there were several constraints, such as complex gene families, the presence of pseudogenes and the lack of a reference genome, that limited the final results. The algorithm designed in this study can address these problems, correctly resolving the gene models. It is also necessary to note is that the guided recovery using genes from model organisms, such as $A$. thaliana, $P$. trichocarpa, $O$. sativa or $P$. patens, could be useful when the coding sequence is incomplete. However, if enough transcript sequence information is available, the model can be built without any reference, which can be justified by taking into account that the contigs used to build the gene model come from the pool of single-hsp contigs. This pool has no defined exon-intron contig structure, and the contigs can be either exon fragments or putative pseudogenes.

The pipeline was also able to provide sequences for the $5^{\prime}$ upstream region of most genes $(85 \%)$, and furthermore, $55 \%$ of the gene models have at least one contig containing 500 to $1000 \mathrm{bp}$ of the regulatory region of the gene. These findings indicate that the pipeline can be tuned to find a substantial portion of the gene promoter regions. These results validate the use of gene capture as a technology to provide information about the promoter region of genes in species where reference genomes are not available. This genomic information can be used in silico studies or to obtain the promoter region for functional studies of a significant number of maritime pine genes.

Exploring the organization of maritime pine gene families Asparagine synthetase plays a specific role in nitrogen assimilation and storage in pine trees $[28,36]$. The data derived from gene capture described in this manuscript revealed that this enzyme is encoded by a small gene family in maritime pine with five members: $A S 1, A S 2$, AS3, AS4 and AS5. We also found their counterparts in the P. taeda genome (Fig. 7). It is remarkable that this approach allowed us to obtain the sequence of a new gene, $A S 5$, which had not been previously identified by transcriptomic analyses. The nucleotide sequences of the five genes (Additional file 9: Figure S5) and the deduced AS polypeptides (Additional file 10: Figure S6) are well conserved, with the highest variability located at the 3' end or the carboxy- terminus of the protein. The AS1, $A S 3, A S 4$ and $A S 5$ genes/proteins are more closely related to each other than to $A S 2$.

Phylogenetically, the angiosperm $A S$ genes can be grouped into two classes: $A S I$ and $A S I I[37,38]$. The $A S$ genes belonging to ASII class generally have 13 introns while the ASI class lacks intron five [38]. A phylogenetic tree was generated using the deduced amino acid sequences of the five $A S$ pine genes from $P$. pinaster and 
P. taeda in addition to the AS sequences from Physcomitrella patens and different angiosperms used in previous $A S$ phylogenetic analysis [38] (Fig. 7). Until now, the gymnosperm $A S$ genes were considered to belong to the $A S$ class II group [39]. But it can be concluded from our phylogenetic analysis that the conifer AS proteins do not belong to either $A S$ class I or class II, which had been previously described for angiosperms. As shown in Fig. 7, the pine AS proteins group together separately from either of the ASI or ASII angiosperm classes. The models that are phylogenetically closer to angiosperms are the class II ASN3 gene from Arabidopsis and the class II Potri.005G075700 gene from poplar, which were included in our comparative study of exon/intron $A S$ gene organization. It has previously been suggested that the angiosperm $A S$ class II genes are closer to the ancestral $A S$ gene [38], and our results support this hypothesis because all conifer $A S$ genes conserve the 13 introns in their structure. Moreover, the conifer $A S$ genes are closer to the bryophyte $P$. patens at sequence level.

The $A S$ BAC clone sequenced in this work contains the genomic sequence of the $A S 1$ gene, but due to the close similarity among the AS1, AS3 and AS5 pine genes (Tables 1 and 2, Fig. 4, Additional file 9: Figure S5 and Additional file 10: Figure S6), the model we obtained using the designed algorithm was predominantly AS3. A thorough examination of the contigs recovered by gene capture is included in Additional file 11: Table S6, and it illustrates that numerous contigs could be included in the AS3 instead of the AS1 model in this case. There

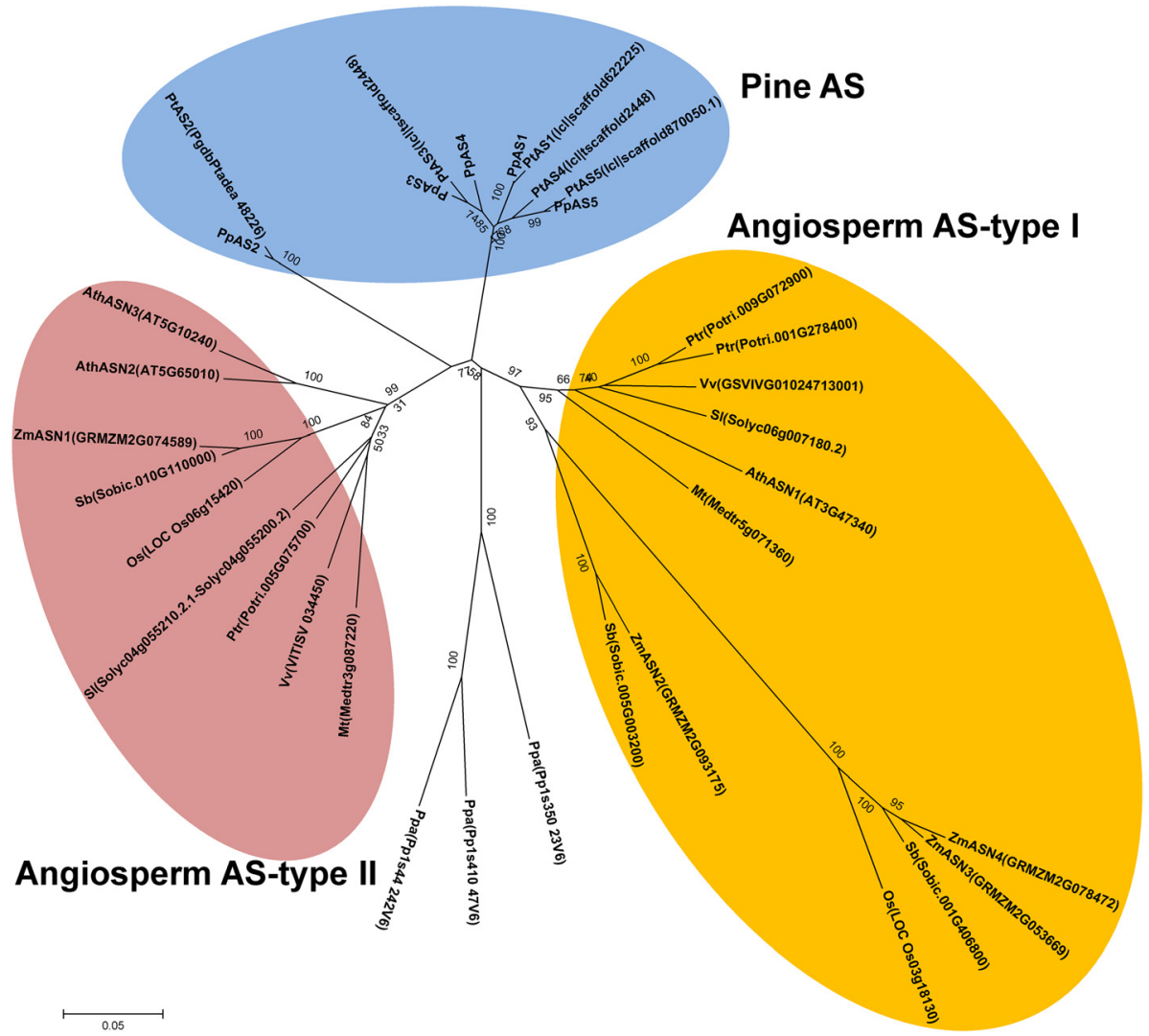

Fig. 7 Phylogenetic tree of the deduced protein sequences of plant genes encoding asparagine synthetase (AS). The optimal tree with the sum of branch length $=2.28234152$ is shown. The tree is drawn to scale, with branch lengths in the same units as those of the evolutionary distances used to infer the phylogenetic tree. The following representative members of the asparagine synthetase (AS) are included in the tree: Arabidopsis thaliana [AthASN1, Phytozome:AT3G47340; AthASN2, Phytozome:AT5G65010; AthASN3, Phytozome:AT5G10240), Medicago truncatula [Phytozome:Medtr5g071360; Phytozome:Medtr3g087220], Oryza sativa [Phytozome:LOC_Os06g15420; Phytozome:LOC_Os03g18130], Physcomitrella patens [Phytozome:Pp1s410_47V6; Phytozome:Pp1s350_23V6; Phytozome:Pp1s44_242V6], Pinus pinaster [AS1, GenBank:ADU02856; AS2, Genbank:ADK13052; AS3, PGC:geneCapture_all_rep_C7631; AS4, SPDB:sp_v3.0_unigene97582/sp_v3.0_unigene8248; AS5, PGC:geneCapture_all_rep_c8956/geneCapture_all_rep_c1052], Pinus taeda [PtAS1, Congenie:Ill|scaffold622225; PtAS2, Congenie:PgdbPtadea_48226; PtAS3, Congenie:Ill|tscaffold2448; PtAS4, Congenie:Icl|tscaffold2448; PtAS5, Congenie:Icl|scaffold870050.1], Populus trichocarpa [Phytozome:Potri.005G075700; Phytozome:Potri.009G072900; Phytozome:Potri.001G278400], Sorghum bicolor [Phytozome:Sobic.005G003200; Phytozome:Sobic.001G406800; Phytozome:Sobic.010G1 10000], Solanum lycopersicum [Phytozome:Solyc06g007180.2; Phytozome:Solyc04g055210.2.1- Phytozome:Solyc04g055200.2], Vitis vinifera [Phytozome:GSVIVG01024713001; Phytozome:VITISV_034450], Zea mays [ZmASN1, Phytozome:GRMZM2G074589; ZmASN2, Phytozome:GRMZM2G093175; ZmASN3, Phytozome:GRMZM2G053669; ZmASN4, Phytozome:GRMZM2G078472] 
were mainly truncated sequences, or intron-lacking sequences, that can be considered to be pseudogenes of $A S 3$, and this indicates that the presence of numerous pseudogenes derived from a single gene can bias the results of this type of gene capture experiment. In the early stages of our study, we included this gene model because we thought it would be a good example of a pine gene belonging to a family with relatively few members to the XET gene and its family composition. In addition, the $A S$ gene has a relatively large number of exons compared to the structure of the XET gene, which has fewer exons that can be used to detect differences in sequence recovery. However, we have found that this additional information related to the numerous pseudogenes in the $A S$ family highlights the complexity of the maritime pine genome.

\section{Conclusions}

The comparison of the gene models produced by both approaches revealed that (i) processing the gene capture data with GeneAssembler was highly successful with $82 \%$ of the gene models recovered even when a reference genome was not available. (ii) Successful and useful gene models can be obtained using probes designed from cDNAs; inference from of a single gene model would be limited in complex gene families with many members for which supplementary information would be required. (iii) Gene capture can serve to fill gaps in the gene structure established by BAC sequencing, as is the case with the SuSy gene model included in this study. (iv) Studying the gene models of different members belonging to the same family (e.g., $A S$ ) creates new possibilities for facilitating intra- and inter- comparative studies aimed at understanding function in the light of evolution.

\section{Methods}

\section{PCR-based screening of a pooled BAC library}

A $0.8 \mathrm{x}$ coverage pooled BAC library was previously prepared [22] as 83 glycerol stocks of E. coli pools, and each pool contained approximately 4000 distinct clones with an average size of $107 \mathrm{kbp}$. DNA of BAC pool was prepared by a modified alkaline lysis method using a previously described protocol [40].

For the primary PCR screening, specific genomic sequences of asparagine synthetase $(A S)$, sucrose synthase $(\mathrm{SuSy})$ genes or xyloglucan endotransglycosylase (XET) were amplified using primers designed against the available cDNA sequence (Additional file 12: Table S5). The PCR profile used for screening the pool library was as follows: $20 \mathrm{~s}$ denaturing at $94{ }^{\circ} \mathrm{C}, 45 \mathrm{~s}$ annealing at $60^{\circ} \mathrm{C}$ and $45 \mathrm{~s}$ elongation at $72{ }^{\circ} \mathrm{C}$. This temperature profile was repeated for 35 cycles, and putative positive PCR product pools were on sequenced a CEQ 8000 automated capillary sequencer (Beckman Coulter, Barcelona Spain). After this, the original cell pools were titrated to determine the appropriate dilution to obtain $\sim 7000$ colonies per Qtrays Genetix plate $(24.2 \times 24.2 \mathrm{~cm})$, and the different clones were then individualized in $36 \times 384$-well plates using a QPIX2 ${ }^{\circ}$ (Genetix).

For the secondary screening, high-density filters were prepared on $22.2 \times 22.2$-cm nylon membranes using a 96pin gridding head on the Genetix QPIX2 ${ }^{\circ}$ robot. Each BAC filter was gridded to 6 rows $\times 4$ columns in a single spot and distributed in six different fields. Membranes were incubated overnight at $37^{\circ} \mathrm{C}$ with the colony side facing up on $\mathrm{LB} / \mathrm{chloramphenicol}(12.5 \mu \mathrm{g} / \mathrm{ml})$ plates, and processing and hybridization of the filters was done as recommended in the QPix manual. $\left[{ }^{32} \mathrm{P}\right]$-labeled specific genomic sequences were used as probes. The hybridized membranes were exposed to a phosphorimaging screen (Fuji Imaging plate BAS-MS 2040) for $24 \mathrm{~h}$ at room temperature and scanned using an FLA-7000 Imaging System (Fujifilm). Using a grid map, the pattern of hybridization allowed us to identify the 384-well plate(s) and the plate address(es) of the positive clone(s).

For the subsequent steps, the cells of the positive clones were recovered and plated on LB agar with $12.5 \mu \mathrm{g} / \mathrm{ml}$ chloramphenicol, $90 \mu \mathrm{g} / \mathrm{ml}$ IPTG and $90 \mu \mathrm{g} /$ $\mathrm{ml} \mathrm{X-GAL}$. After a second round of PCR and sequencing, the presence of a specific genomic sequence was confirmed. The detailed workflow protocol is available in Additional file 1: Figure S1.

\section{BAC DNA Sequencing library preparation and 454 parallel sequencing}

The BAC insert size was estimated by pulse field gel electrophoresis (FIGE, Bio-rad Lab. Inc.). The 454 sequencing library was prepared with $6 \mu \mathrm{g}$ of the BAC plasmid DNA using shotgun and standard long, pairedend sequencing kits, according to the specifications of Roche, the manufacturer. Sequencing libraries were quantified with 2100 BioAnalyzer (Agilent), processed by emulsion PCR and sequenced on a 454/Roche GS FLX as described in the manuals (Roche Diagnostics). The 24 L12 (AS), 25 M3 (XET) BACs libraries, were respectively sequenced in paired-end and shotgun pools using MIDs loaded in a $1 / 2$ region, and BAC $26 \mathrm{C} 15$ library ( SuSy) was sequenced in paired-end pools in a $1 / 4$ region of $70 \times 75$ Picotiterplate (PTP). The pair-end BAC reads, which had been preprocessed by SeqTrimNext, were assembled using Newbler version 2.3 with the default parameters.

\section{Capture array gene selection}

The 91,086 full-length unigenes predicted from the $P$. pinaster transcriptome assembly from SustainPineDB [20] were used to select the genes for the DNA capture 
array. The full-length unigenes were filtered based on their GO terms [41], and the KEGG pathways [42] annotations mainly focused in metabolism. Because some unigenes contained annotations from several functions, this filter resulted in 1462 unigenes. Additionally, to reduce redundancy, the unigenes sharing the same ortho$\log$ from UniProt [43] were filtered to get only one gene per ortholog, reducing the selected number of unique unigenes to 1026. BLAST [44] best hits were used to assign the orthologous genes.

After the unigene selection was done, a better assembly of $P$. pinaster transcriptome was performed and published [20], so we searched for the genes from the 1026 unigenes in the SustainPine assembly version 1.1 (http:// www.scbi.uma.es/sustainpinedb/) using BLAST. Finally, 866 unigene sequences were selected to be included in the DNA capture array. As described previously, we added 3 genomic sequences from cloned BACs as control sequences, the asparagine synthetase [BAC at GenBank: KP172187] from 5' UTR to the stop codon, a clone containing the sequence of a gene encoding a sucrose synthase [BAC at GenBank: KP172194] and a clone containing the xyloglucan endotransglycosylase [BAC at GenBank: KP172185]. The distribution of the unigenes used for the capture array in functional categories is shown in Additional file 13: Figure S7.

\section{DNA capture array design}

A Ruby custom script was used to design the probes for an Agilent SureSelect DNA Capture Array, and it was optimized to fit the 866 genes selected (see Capture array gene selection). In total, 56,667 probes of $120 \mathrm{nt}$ in length were designed, and the probe density was increased in the first third of the transcript sequences (including the 5'UTR). In this way, all of the genes were represented in the array with $6 \mathrm{x}$ coverage in the first third of the gene sequence (using a tilling distance of $19 \mathrm{nt}$ ) and $4 \mathrm{x}$ coverage in the other two thirds (using a tilling distance of $29 \mathrm{nt}$ ). On average, 65 overlapping probes represented each gene, and they were uploaded to the Agilent eArray website (https://earray.chem.agilent.com/earray/) for the production of the array.

\section{Haploid genome DNA preparation}

Maritime pine cones were collected from Oria 6, a genotype of $P$. pinaster Aiton from the natural population Sierra de Oria (Almería, Spain), selected based on its response to extreme drought conditions. Cones were surface sterilized with $96 \%$ ethanol for $20 \mathrm{~min}$ and airdried in a laminar flow cabinet before seed isolation. Haploid megagametophytes were isolated from sterilized seeds for tissue culture establishment [25]. For sequence capture, the $P$. pinaster A5 callus haploid line derived from the megagametophyte tissue was used. The DNA was extracted from the calli using a DNeasy Plant Mini Kit (Qiagen) and it was quantified with a Quant-it PicoGreen dsDNA Assay Kit (Invitrogen). The quality was assessed with an Agilent 2100 Bioanalyzer.

\section{Targeted capture and $\mathbf{4 5 4}$ parallel sequencing}

Haploid genomic DNA from $P$. pinaster was captured using the Agilent SureSelect Target Enrichment System following the manufacturer's protocols with minor modifications. Two micrograms of this DNA were fragmented to $1.5 \mathrm{~kb}$ in size and purified by gel extraction using a MinElute Gel extraction kit (Qiagen), and the quality of fragmentation and purification was assessed with an Agilent 2100 Bioanalyzer. Fragment ends were repaired, and RL adaptors (Roche) were ligated to the fragments, and the resulting adapter-ligated sample was purified using Agencourt AMPure XP beads (Beckmann Coulter). The DNA library was amplified by PCR and captured by hybridization at $65{ }^{\circ} \mathrm{C}$ for $24 \mathrm{~h}$ with the biotinylated RNA library "bait" (Agilent). Bound genomic DNA was purified with streptavidin-coated magnetic Dynabeads (Invitrogen) and re-amplified. Stratagene Herculase II enzyme (Agilent) was used for both PCR reactions, and the resulting captured library was purified using Agencourt AMPure XP beads (Beckmann Coulter) and was assessed with the Agilent 2100 Bioanalyzer. Finally the captured library was sequenced on Roche GS-FLX+ using a two-region gasket according to the manufacturer's protocols.

\section{Roche $\mathbf{4 5 4}$ data processing}

The reads were preprocessed using the SeqTrimNext pipeline (http://www.scbi.uma.es/seqtrimnext) [45], which is available from the Plataforma Andaluza de Bioinformatica (University of Malaga, Spain). Low quality sequences, linkers, adaptors, vector fragments, organelle DNA, and contaminated sequences were removed, and the longest informative part of the read was retained, discarding sequences below $40 \mathrm{bp}$.

\section{Data assembly and gene recovery criteria}

Useful reads were assembled by the MIRA assembler, version 3 [46], to obtain the contigs for building the gene models. Full-length transcripts and the protein sequences were predicted using Full-Lengther Next [47] based on SustainPine 1.1 database [20] information.

The captured fragments were identified, and the gene models were generated using our own bioinformatic pipeline (GeneAssembler), which can be downloaded and installed on any Unix/Linux-based computer as a Ruby gem: https://rubygems.org/gems/gene_assembler (for a detailed description of the GeneAssembler pipeline see Additional file 14). 
The gene sequences from Arabidopsis thaliana, Populus trichocarpa, Oryza sativa and Physcomitrella patens were downloaded from Phytozome 9.1 [48] to improve gene recovery, and an ortholog search was performed to enhance the gene model building strategy. A Blast X (by default parameters) [49] with the full-length proteins was performed against each set of genes. All gene matches were considered to be putative orthologs, which means that for each match, the exon-intron coordinate was retrieved.

\section{Sequence alignment and phylogenetic analysis}

The sequences used for alignments and phylogenetic trees were obtained in Phytozome database (http://phytozome.jgi.doe.gov) except for $P$. taeda that were obtained from Congenie database (http://congenie.org/). P. pinaster AS1 [GenBank:ADU02856]; and AS2 [GenBan$\mathrm{k}: A D K 13052]$ protein sequences were obtained from GenBank at the NCBI. For P. pinaster AS3 [PGC:geneCapture_all_rep_c7631] and AS5 [PGC:geneCapture_all_rep_c8956/geneCapture_all_rep_c10521 we used sequences obtained in the course of this work and deposited in the Pine Gene Capture database (PGC, http:// www.scbi.uma.es $/ \mathrm{pgc} /$ ). Finally for $P$. pinaster AS4 [SPDB: sp_v3.0_unigene97582/sp_v3.0_unigene8248] we used the sequence obtained from our transcriptomic database SustainPineDB [20] (http://www.scbi.uma.es/ sustainpinedb/).

The CLUSTALW program was used for sequence alignments [50]. The phylogenetic tree was constructed with full-length AS amino acid sequences using the neighbor-joining method [51] with 1000 bootstrap replications. The evolutionary distances were computed using the JTT matrix-based method [52] and are in the units of the number of amino acid substitutions per site. The rate variation among sites was modeled with a gamma distribution (shape parameter $=1$ ). All ambiguous positions were removed for each sequence pair. The positions not presented in all the sequences were eliminated. Finally there were a total of 509 positions in the final dataset. All of these analyses were conducted in MEGA6 [53].

\section{Availability of supporting data}

The bioinformatic pipeline (GeneAssembler) used for generating gene models, can be downloaded at: https:// rubygems.org/gems/gene_assembler. All of the generated contigs and models are available in the Pine Gene Capture database (PGC, http://www.scbi.uma.es/pgc/). The phylogenetic data can be found at http://purl.org/phylo/ treebase/phylows/study/TB2:S18787. Other supporting data of this article are included as additional files

\section{Additional files}

\begin{abstract}
Additional file 1: Figure S1. Workflow overview followed to isolate and sequence maritime pine BAC clones. (A) Primary PCR screening of pools of the BAC library using specific oligonucleotides against XET CDNA. (B) Plating of cells of the positive pools on $24.2 \times 24.2 \mathrm{~cm}$ plates. (C) The single clones of the pool were individualized in 36 X 384 plates using a QPix and grown orderly gridded in high-density filters. (D) The replica filters containing the ordered single clones of each pool were hybridized with cDNA ${ }^{32} \mathrm{P}-\mathrm{a}$ beled specific probes and exposed to a phosphorimaging screen. (E) Secondary PCR screening was performed on single clones to isolate positives. (F) BAC DNA isolation of positives to prepare a 454 library either for shotgun or paired-end sequencing. (TIFF $6431 \mathrm{~kb}$ )
\end{abstract}

Additional file 2: Table S1. Exon length comparison between SuSy BAC clone from $P$. pinaster and SuSy from two angiosperm plants. The first exon is lacking in the BAC clone. The gene capture model is also included (DOCX 19 kb)

Additional file 3: Table S2. Intron length comparisons between Susy BAC clone from $P$. pinaster and Susy from two angiosperm plants. The intron 18 in the BAC clone contains a gap. The gene capture model is also included (DOCX $20 \mathrm{~kb})$

Additional file 4: Table S3. Exon length comparison between the XET BAC clone from P. pinaster and two XET genes from Arabidopsis thaliana. The two gene capture models closest to the BAC clone are also included. (DOCX $18 \mathrm{~kb}$ )

Additional file 5: Table S4. Intron length comparison between the XET BAC clone from P. pinaster and two XET genes from Arabidopsis thaliana. The two gene capture models closest to the BAC clone are also included. (DOCX $17 \mathrm{~kb}$ )

Additional file 6: Figure S2. Alignment of the GS1a gene promoter [GenBank:AJ225121], to the gene capture GS1a gene 5'upstream region (TIF $245 \mathrm{~kb}$ )

Additional file 7: Figure S3. Alignment of the PAL gene promoter [GenBank:HE866754], to the gene capture PAL gene 5'upstream region (TIF 389 kb)

Additional file 8: Figure S4. Alignment of the PAT gene promoter [GenBank:HE866755], to the gene capture PAT gene 5'upstream region. (TIF $332 \mathrm{~kb}$ )

Additional file 9: Figure S5. Nucleotide alignment of maritime pine AS CDNAs. (TIF $533 \mathrm{~kb}$ )

Additional file 10: Figure S6. Amino acid alignment of maritime pine AS protein (TIF $460 \mathrm{~kb}$ )

Additional file 11: Table S6. Summary of AS contigs obtained in the gene capture and used to build the AS model. (XLS $138 \mathrm{~kb}$ )

Additional file 12: Table S5. Primers used in PCR for validation of positive BAC clones in the BAC library screening. (DOCX $39 \mathrm{~kb}$ )

Additional file 13: Figure S7. Functional categories by GO terms for the full-length unigenes used in the capture approach (TIF $269 \mathrm{~kb}$ )

Additional file 14: Detailed description of the GeneAssembler pipeline (DOCX $124 \mathrm{~kb})$

Competing interests

The authors declare that they have no competing interests.

\section{Authors' contributions}

PSZ and MGC developed the algorithm for gene capture sequence analysis. RAC conducted the study of the AS family and wrote part of the manuscript. RB analyzed 454 sequences and performed BAC assembly. JGM performed BAC screening and high throughput 454 sequencing. IA obtained the A5 haploid callus line used for genomic DNA extraction. NFP helped in selecting genes and designed probes for targeted capture. MGC contributed to the writing of the bioinformatics part of the manuscript. FMC analyzed data and wrote part of the manuscript. CA coordinated experimental and bioinformatics work, designed the BAC experiments and wrote the manuscript. All authors read and approved the final manuscript. 


\section{Acknowledgments}

This work has been funded by grants from the European Commission Seventh Framework grant PROCOGEN (FP7-KBBE-2011-5) and the Spanish Ministerio de Economía y Competitividad (BIO2012-33797). PSZ was supported by funds from Junta de Andalucía, P10-CVI-6075

\section{Author details}

'Departamento de Biología Molecular y Bioquímica, Facultad de Ciencias, Universidad de Málaga, Campus de Teatinos s/n, E-29071 Málaga, Spain. ${ }^{2}$ Departamento de Biología Vegetal, Facultad de Farmacia, ERI Biotecmed, Universidad de Valencia, Avda. Vicent Andrés Estellés s/n, 46100 Burjassot, Valencia, Spain. ${ }^{3}$ Boyce Thompson Institute for Plant Research, Cornell University, Ithaca, NY 14853, USA.

\section{Received: 28 October 2015 Accepted: 17 February 2016} Published online: 27 February 2016

\section{References}

1. Olson JS, Watts JA, Allison LJ. Carbon in live vegetation of major world ecosystems. In: Report ORNL. Oak Ridge: Oak Ridge National Laboratory; 1983. p. 5862.

2. Farjon A. A Handbook of the World's Conifers. Leiden: Brill Academic Publishers; 2010

3. Hamrick JL, Godt MJW, Sherman-Broyles SL. Factors influencing levels of genetic diversity in woody plant species. New For. 1992;6:95-124.

4. Mackay J, Dean JFD, Plomion C, Peterson DG, Cánovas FM, Pavy N, et al. Towards decoding the conifer giga-genome. Plant Mol Biol. 2012;80:555-69.

5. Nystedt B, Street NR, Wetterbom A, Zuccolo A, Lin Y-C, Scofield DG, et al. The Norway spruce genome sequence and conifer genome evolution. Nature. 2013;497:579-84.

6. Birol I, Raymond A, Jackman SD, Pleasance S, Coope R, Taylor GA, et al. Assembling the $20 \mathrm{~Gb}$ white spruce (Picea glauca) genome from wholegenome shotgun sequencing data. Bioinformatics. 2013;29:1492-7.

7. Wegrzyn JL, Lin BY, Zieve JJ, Dougherty WM, Martinez-Garcia PJ, Koriabine $\mathrm{M}$, et al. Insights into the Loblolly Pine Genome: Characterization of BAC and Fosmid Sequences. PLoS One. 2013:8:e72439.

8. de la Torre AR, Birol I, Bousquet J, Ingvarsson PK, Jansson S, Jones SJM, et al. Insights into conifer Giga-Genomes. Plant Physiol. 2014;166:1724-32.

9. Kovach A, Wegrzyn JL, Parra G, Holt C, Bruening GE, Loopstra CA, et al. The Pinus taeda genome is characterized by diverse and highly diverged repetitive sequences. BMC Genomics. 2010;11:420.

10. Lander ES, Consortium IHGS, Linton LM, Birren B, Nusbaum C, et al. Initial sequencing and analysis of the human genome. Nature. 2001;409:860-921.

11. Rampant PF, Lesur I, Boussardon C, Bitton F, Martin-Magniette ML, Bodenes $C$, et al. Analysis of BAC end sequences in oak, a keystone forest tree species, providing insight into the composition of its genome. BMC Genomics. 2011;12:292.

12. Sena JS, Giguère I, Boyle B, Rigault P, Birol I, Zuccolo A, et al. Evolution of gene structure in the conifer Picea glauca: a comparative analysis of the impact of intron size. BMC Plant Biology. 2014;14:95.

13. Mamanova L, Coffey AJ, Scott CE, Kozarewa I, Turner EH, Kumar A, et al. Target-enrichment strategies for next-generation sequencing. Nat Methods. 2010;7:111-8.

14. Lin X, Tang W, Ahmad S, Lu J, Colby CC, Zhu J, et al. Applications of targeted gene capture and next-generation sequencing technologies in studies of human deafness and other genetic disabilities. Heart Research. 2012;288:67-76

15. Choi M, Scholl UI, Ji W, Liu T, Tikhonova IR, Zumbo P, et al. Genetic diagnosis by whole exome capture and massively parallel DNA sequencing. PNAS. 2009;106:19096-101.

16. Ng SB, Turner EH, Robertson PD, Flygare SD, Bigham AW, Lee C, et al. Targeted capture and massively parallel sequencing of 12 human exomes. Nature. 2009;461:272-6.

17. Bamshad MJ, Ng SB, Bigham AW, Tabor HK, Emond MJ, Nickerson DA, et al. Exome sequencing as a tool for Mendelian disease gene discovery. Nat Rev Genet. 2011;12:745-55

18. Neves LG, Davis JM, Barbazuk WB, Kirst M. Whole-exome targeted sequencing of the uncharacterized pine genome. Plant J. 2013;75:146-56.

19. Mascher M, Richmond TA, Gerhardt DJ, Himmelbach A, Clissold L, Sampath $D$, et al. Barley whole exome capture: a tool for genomic research in the genus Hordeum and beyond. Plant J. 2013;76:494-505.
20. Canales J, Bautista R, Label P, Gomez-Maldonado J, Lesur I, Fernandez-Pozo $\mathrm{N}$, et al. De novo assembly of maritime pine transcriptome implications for forest breeding and biotechnology. Plant Biotechnol J. 2014;12(3):286-99.

21. Cañas RA, Canales J, Gómez-Maldonado J, Ávila C, Cánovas FM. Transcriptome analysis in maritime pine using laser capture microdissection and 454 pyrosequencing. Tree Physiol. 2014;34:1278-88.

22. Bautista R, Villalobos DP, Díaz-Moreno S, Cantón FR, Canovas FM, Claros MG. Toward a Pinus pinaster bacterial artificial chromosome library. Ann Forest Sci. 2007;64:855-64

23. Fernández-Pozo N, Guerrero-Fernández D, Bautista R, Gómez-Maldonado J, Avila C, Cánovas FM, et al. GENote v. $\beta$ :: A web tool prototype for annotation of unfinished sequences in non-model Eukaryotes. In: Freitas AT, Navarro A, editors. Lecture Notes in Bioinformatics. Berlin: Springer; 2012. p. 66-71.

24. Canales J, Rueda-López M, Craven-Bartle B, Avila C, Cánovas FM. Novel insights into regulation of asparagine synthetase in conifers. Frontiers Plant Sci. 2012;3:100.

25. Arrillaga I, Guevara MA, Muñoz-Bertomeu, Lázaro-Gimeno D, Sáez-Laguna E, Díaz LM, et al. Selection of haploid cell lines from megagametophyte cultures of maritime pine as a DNA source for massive sequencing of the species. Plant Cell Tiss Organ Cult. 2014;118:147-55.

26. Petrov DA. Evolution of genome size: new approaches to an old problem. Trends Genet. 2001;17:23-8.

27. Wendel JF, Cronn RC, Alvarez I, Liu B, Small RL, Senchina DS. Intron size and Genome size in plants. Mol Biol Evol. 2002;19:2346-52.

28. Cañas RA, de la Torre F, Cánovas FM, Cantón FR. High levels of asparagine synthetase in hypocotyls of pine seedlings suggest an essential role of the enzyme in re-allocation of seed-stored nitrogen. Planta. 2006;224:83-95.

29. Tuskan GA, Difazio S, Jansson S, Bohlmann J, Grigoriev I, Hellsten U, et al. The genome of black cottonwood, Populus trichocarpa (Torr. \& Gray). Science. 2006;313:1596-604

30. Gómez-Maldonado J, Avila C, Barnestein P, Crespillo R, Cánovas FM. Interaction of cis-acting elements in the expression of a gene encoding cytosolic glutamine synthetase in pine seedlings. Physiol Plant. 2004;121:537-45.

31. Craven-Bartle B, Pascual MB, Cánovas FM, Avila C. A Myb transcription factor regulates genes of the phenylalanine pathway in maritime pine. Plant J. 2013;74:755-66.

32. Bennetzen JL. Mechanisms and rates of genome expansion and contraction in flowering plants. Genetica. 2002;115:29-36.

33. Walbot V, Petrov DA. Gene galaxies in the maize genome. Proc Natl Acad Sci. 2001;98:8163-4.

34. Morse AM, Peterson DG, Islam-Faridi MN, Smith KE, Magbanua Z, Garcia SA, et al. Evolution of Genome size and complexity in Pinus. PloS One. 2009:4:e4332

35. Magbanua ZV, Ozkan S, Bartlett BD, Chouvarine P, Saski CA, Liston A, et al. Adventures in the Enormous: A 1.8 Million Clone BAC library for the $21.7 \mathrm{~Gb}$ Genome of loblolly Pine. PloS One. 2011;6:e16214.

36. Cánovas FM, Avila C, Cantón FR, Cañas RA, de la Torre F. Ammonium assimilation and amino acid metabolism in conifers. J Exp Bot. 2007;58: 2307-18.

37. Herrera-Rodríguez MB, Carrasco-Ballesteros S, Maldonado JM, Pineda M, Aguilar M, Pérez-Vicente R. Three genes showing distinct regulatory patterns encode the asparagine synthetase of sunflower (Helianthus annus). New Phytol. 2002:155:33-45.

38. Cañas RA, Quilleré I, Christ A, Hirel B. Nitrogen metabolism in the developing ear of maize (Zea mays): analysis of two lines contrasting in their mode of nitrogen management. New Phytol. 2009;184:340-52.

39. Gaufichon L, Reisdorf-Cren M, Rothstein SJ, Chardon F, Suzuki A. Biological functions of asparagine synthetase in plants. Plant Sci. 2010;179:141-53.

40. Villalobos D, Bautista R, Cánovas FM, Claros MG. Isolation of Bacterial Artificial Chromosome DNA by Means of Improved Alkaline Lysis and Double Potassium Acetate Precipitation. Plant Mol Biol Rep. 2004;22:1-7.

41. Barrell D, Dimmer E, Huntley RP, Binns D, O'Donovan C, Apweiler R. The GOA database in 2009-an integrated Gene Ontology Annotation resource. Nucleic Acids Res. 2009:37(Database issue):D396-403.

42. Kanehisa M, Goto S, Sato Y, Kawashima M, Furumichi M, Tanabe M. Data, information, knowledge and principle: back to metabolism in KEGG. Nucleic Acids Res. 2014;42(Database issue):D199-205.

43. UniProt Consortium. Activities at the Universal Protein Resource (UniProt). Nucleic Acids Res. 2014;42(Database issue):D191-8.

44. Altschul SF, Gish W, Miller W, Myers EW, Lipman DJ. Basic local alignment search tool. J Mol Biol. 1990;215(3):403-10. 
45. Falgueras J, Lara AJ, Fernandez-Pozo N, Canton FR, Perez-Trabado G, Claros MG. SeqTrim: a high-throughput pipeline for pre-processing any type of sequence read. BMC Bioinformatics. 2010;11:38.

46. Chevreux B, Pfisterer T, Drescher B, Driesel AJ, Muller WE, Wetter T, et al. Using the miraEST assembler for reliable and automated mRNA transcript assembly and SNP detection in sequenced ESTs. Genome Res. 2004;14(6):1147-59.

47. Lara A, Pérez-Trabado G, Villalobos D, Díaz-Moreno S, Cantón F, Claros MG. A Web Tool to Discover Full-Length Sequences: Full-Lengther. In: Corchado E, Corchado JM, Abraham A, editors. Innovations in Hybrid Intelligent Systems. Berlin: Springer; 2007. p. 361-8.

48. Goodstein DM, Shu S, Howson R, Neupane R, Hayes RD, Fazo J, et al. Phytozome: a comparative platform for green plant genomics. Nucleic Acids Res. 2012;40(Database issue):D1178-86.

49. Camacho C, Coulouris G, Avagyan V, Ma N, Papadopoulos J, Bealer K, et al. BLAST+: architecture and applications. BMC Bioinformatics. 2009;10:421. doi: 10.1186/1471-2105-10-421.

50. Thompson JD, Higgins DG, Gibson TJ. CLUSTAL W: improving the sensitivity of progressive multiple sequence alignment through sequence weighting, position-specific gap penalties and weight matrix choice. Nucleic Acids Res. 1994;22:4673-80.

51. Saitou N, Nei M. The neighbor-joining method: A new method for reconstructing phylogenetic trees. Mol Biol Evol. 1987;4:406-25.

52. Jones DT, Taylor WR, Thornton JM. The rapid generation of mutation data matrices from protein sequences. Comput Appl Biosci. 1992;8:275-82.

53. Tamura K, Stecher G, Peterson D, Filipski A, Kumar S. MEGA6: Molecular Evolutionary Genetics Analysis version 6.0. Mol Biol Evol. 2013;30:2725-9.

\section{Submit your next manuscript to BioMed Central and we will help you at every step:}

- We accept pre-submission inquiries

- Our selector tool helps you to find the most relevant journal

- We provide round the clock customer support

- Convenient online submission

- Thorough peer review

- Inclusion in PubMed and all major indexing services

- Maximum visibility for your research

Submit your manuscript at www.biomedcentral.com/submit 